\title{
Disseminated zygomycosis associated with erythroleukaemia: confirmation by lectin stains
}

\author{
EW BENBOW, IW DELAMORE, ${ }^{*}$ RW STODDART, H REID \\ From the Departments of Pathology and ${ }^{*}$ Haematology, University of Manchester, Manchester
}

SUMmaRY Zygomycosis is not often diagnosed in the United Kingdom, and so the possible importance of the findings in a patient with disseminated zygomycosis who had been treated with chemotherapy for erythroleukaemia was not appreciated until histological examination of specimens obtained at necropsy provided a presumptive diagnosis. No attempt had therefore been made to identify the organism by culture, and lectin binding methods were used to try to compensate for this. The characteristics of the hyphae on staining with lectins were similar to those previously shown in Rhizopus oryzae and were unlike those of a wide range of other hyphal fungi. Although definite speciation of the fungus was not achieved, these findings confirm that this was a case of zygomycosis and would seem to represent the first such reported confirmation in the absence of culture.

Six principal patterns of infection of an organ with zygomycosis are recognised: rhinocerebral, pulmonary, disseminated, gastrointestinal, cutaneous, and miscellaneous. ${ }^{1-3}$ Each pattern of infection is associated with a characteristic range of underlying conditions, with a particularly prominent and well documented association between diabetes mellitus and rhinocerebral zygomycosis. ${ }^{245}$ There is also a strong association between haematological malignancy and the development of the disseminated form of zygomycosis. ${ }^{67}$ Various other predisposing factors have been well reviewed by McNulty, ${ }^{8}$ and several cases have occurred in previously apparently well subjects.

Opportunistic infections caused by organisms of the order Mucorales are confusingly named, for the terms mucormycosis, phycomycosis, and zygomycosis are often used as if they were interchangeable. Terminology of disease has clearly failed to keep up with changes in taxonomy. Phyomycosis is often used, though taxonomic changes subsequent to its introduction seem to ren-

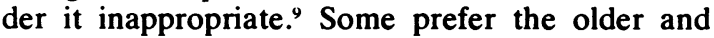
more familiar term mucormycosis for infections diagnosed by biopsy without identification of the exact species by culture, ${ }^{10}$ although the term has been abandoned by the nomenclature committee of the International Society for Human and Animal Mycology." Zygomycosis has the advantage of

Accepted for publication 16 May 1985 reflecting the current name of the class. ${ }^{12}$ Most deep infections ascribable to Zygomycetes are caused by members of the genera Rhizopus, Mucor, and Absidia. $^{3}$

We saw a case of disseminated mucormycosis in a woman with leukaemia. The presence of opportunistic fungal infection was not suspected during life and was not detected until necropsy. Conventional special stains were supplemented with lectin staining because of the ability of lectins to stain identifiable carbohydrate residues. The high saccharide content of fungal walls suggests that lectin staining may be of possible value in detecting fungi in histological sections. ${ }^{13}$

\section{Case report}

A woman aged 66 was transferred to Manchester Royal Infirmary in September 1981. She complained of lethargy, malaise, oral ulceration, and dysphagia. Peripheral blood counts were as follows: Haemoglobin concentration $7.3 \mathrm{~g} / \mathrm{dl}$; white cell count $120.0 \times 10^{9} / 1$ (98\% blasts, $2 \%$ lymphocytes); and platelets $36 \times 10^{9} / 1$. Bone marrow smears showed complete replacement of the normal bone marrow cells by myeloblasts. Special stains confirmed the diagnosis of acute myeloblastic leukaemia.

She was treated with a combination of daunorubicin, cytosine arabinoside, and thioguanine, according to the protocol laid down by the Medical Research Council's 8th acute myeloblastic leu- 
kaemia trial. Remission was confirmed by repeat examination of bone marrow after two courses of chemotherapy. She was given two consolidation courses with the same combination of drugs, and after she had been in remission for one year late intensification treatment was started. This consisted of four courses of treatment separated by intervals of three weeks; each course included cyclophosphamide $900 \mathrm{mg}$ and vincristine $200 \mathrm{mg}$ intravenously at once, cytosine arabinoside subcutaneously twice daily for five days, and prednisolone $100 \mathrm{mg}$ orally each day for five days, with chlorpromazine $25 \mathrm{mg}$ orally thrice daily for control of nausea if required.

In early October 1983 a falling peripheral blood count heralded a relapse of leukaemia. The morphological pattern of the bone marrow smear was now that of erythroleukaemia, and further chemotherapy with daunorubicin, cytosine arabinoside, and thioguanine was given. She became pancytopenic, and during this period she developed inflammatory changes in the left orbit. She complained of severe pain and throbbing in the eye and was found to be slightly feverish. Flucloxacillin and benzylpenicillin were given intravenously, together with chloramphenicol eye drops.

When a swab from the eye was reported to have grown coagulase negative staphylococci the intravenous antibiotics were changed to pipericillin and tobramycin. A subsequent report that Streptococcus milleri had been grown from blood cultures led to the substitution of benylpenicillin for pipericillin. By this stage, however, severe proptosis of the left eye had occurred, and the conjunctiva was reported as looking chemotic and necrotic (Fig. 1).
Cerebral angiography was hampered by patient movement and a poor cardiac output, but the internal carotid arteries appeared to be normally patent; the venous phase was not visualised. Because of her persistent fever and continuing deterioration granulocyte transfusions were given, but she died in March 1984.

\section{FINDINGS AT NECROPSY}

External examination showed moderate jaundice, with extensive ecchymoses and mild bilateral ankle oedema. The left periorbital tissues were greatly swollen and showed a deep red discolouration similar to that after fairly recent bruising.

Examination of the external surface of the brain showed pronounced softening and discolouration of the frontal lobes; this was more extensive on the left, where it affected a conical zone of about $4 \mathrm{~cm}$ in depth at the anterior pole of the brain (Fig. 2). The cut surface in this area was friable and was a deep pink colour. The intracranial portion of the left internal carotid artery contained firm thrombus, which extended into the left anterior cerebral artery.

The heart weighed $480 \mathrm{~g}$, with left ventricular hypertrophy. The myocardium appeared pale and flabby but contained no focal lesion. Coronary atheroma was generally of only minor degree. The lungs were focally firm, especially in the upper lobes.

There was an annular ulcer at the lower end of the oesophagus, which measured $3.5 \mathrm{~cm}$ in the long axis? of the viscus. The margins of this lesion were a little thickened, but did not appear to extend through the wall. The liver contained many nodules, ranging in diameter from 0.3 to $1.2 \mathrm{~cm}$, each with a homogenous yellowish cut surface. The intervening hepatic

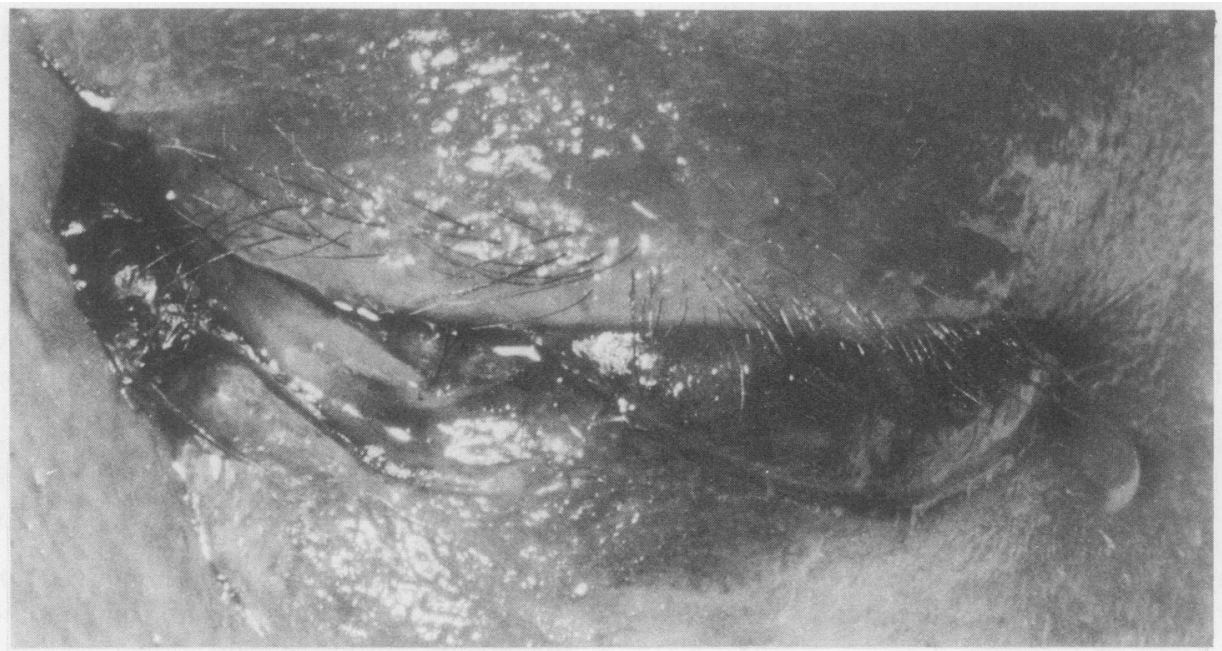

Fig. 1 Left eye, one week before death. 


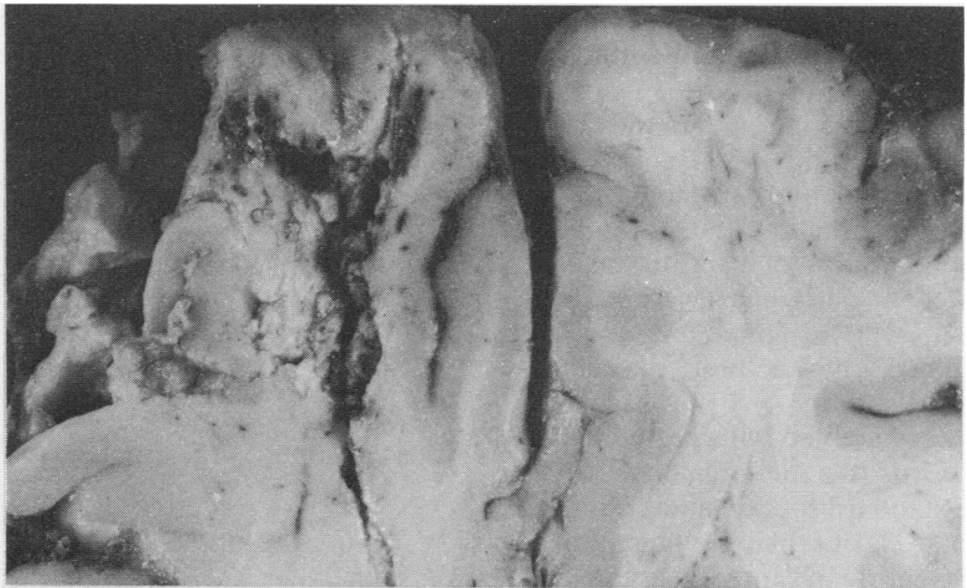

Fig. 2 Part of horizontal slice through fixed brain. Lesions are present in both frontal lobes.

parenchyma was rather pale. The spleen bore an irregular pale lesion measuring $2.0 \times 1.5 \times 1.5 \mathrm{~cm}$ on its diaphragmatic aspect, together with many tiny nodules within the parenchyma. The kidneys showed particularly prominent vascular markings but no other macroscopic abnormality. The other organs appeared unremarkable.

\section{CONVENTIONAL HISTOLOGY}

The frontal lobes were infarcted, and many blood

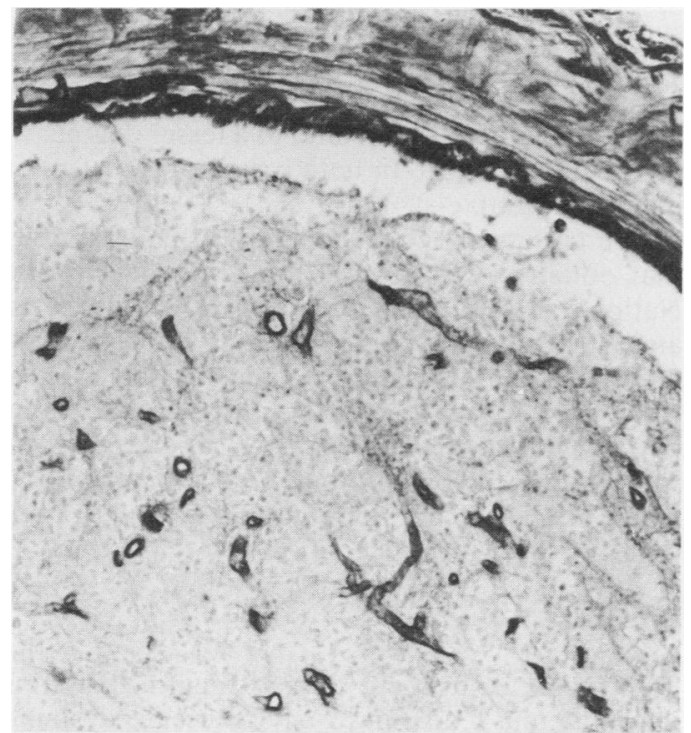

Fig. 3 Hyphae within thrombus in lumen of an intracerebral vessel. Some are also present in the wall. (Grocott stain.) Original magnification $\times 40$. vessels in the abnormal areas and in the adjacent meninges contained fungal hyphae. The hyphae could be seen penetrating vessel walls (Fig. 3) and were visible within infarcted brain tissue. Hyphal diameter was variable, branching was irregular (Fig. 4), and septa were sparse. Indeed, many of the apparent cross walls were pseudosepta formed by kinking of the hyphae. The density of hyphal eosinophilia seemed to vary from area to area.

The hepatic nodules comprised liver plates, show-

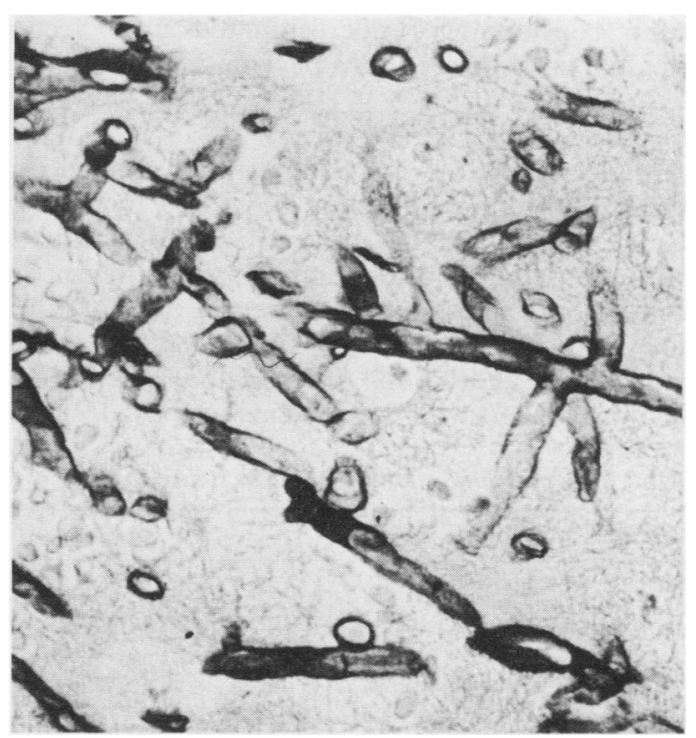

Fig. 4 Fungal hyphae of variable diameter, with irregular branching and pseudoseptae. Left internal carotid artery. (Grocott stain.) Original magnification $\times 170$. 
ing coagulative necrosis with infiltration by fungal hyphae. The splenic lesions were similarly formed of fungal infiltrate, with a rather more regular arrangement of hyphae than that found in the brain, within a background of necrotic tissue. Tiny fungal colonies were present in the kidneys. Sections of the lower oesophagus showed shallow ulceration with an overlying pseudomembrane of necrotic mucosa and fibrinous exudate; fungal hyphae were present only in the superficial layers of this membrane. No evidence of fungal infiltration was found in sections of the heart or lungs.

Selected sections were stained with periodic acid Schiff, Gram, and Grocott's methenamine silver stains. Each of these standard special stains outlined the fungus with greater clarity than the haematoxylin and eosin stain, especially in sites such as the liver and within the thrombus, where the degree of eosinophilia of the hyphae resembled that of the adjacent tissue.

The value of the Gram stain varied between tissues. The hyphae stained well with the magenta counterstain and were particularly clearly defined in the spleen. Unfortunately, the Gram stain appeared to enhance the fibrin strand artefact seen in a blood clot and was therefore of little value in defining the extent of fungus within blood vessels. Periodic acid Schiff and Grocott's stains were of more value and clearly defined the features and extent of fungal infection in all the affected tissues. Indeed, though the presence of fungus had been detected in sections stained with haematoxylin and eosin, its extent had been considerably underestimated until sections treated with special stains became available.

\section{LECTIN STAINS}

A panel of lectin stains (Table) were applied using the avidin-biotin technique described by $\mathrm{Hsu}$ and Raine, ${ }^{14}$ modified by the exclusion of incubation with mouse liver powder and by using different concentrations of reagents to achieve a ratio of $1: 4$ for the components of the peroxidase complex labelled with avidin and biotin. ${ }^{15}$ In addition, a selection of lectins labelled with fluorescein were used. ${ }^{13}$

Table Identity and affinities of lectin stains applied

\begin{tabular}{ll}
\hline Lectin & Affinity \\
\hline Wheatgerm agglutinin & +++ \\
Concanavalin $\mathrm{A}^{*}$ & - \\
Garden pea agglutinin & + \\
Lentil agglutinin & $+1-$ \\
Erythrophytohaemagglutinin & ++ \\
Soy bean agglutinin & - \\
Peanut agglutinin & - \\
Winged pea agglutinin & + \\
\hline
\end{tabular}

*Concanavalin $\mathbf{A}$ and soy bean agglutinin staining is difficult to quantify because of staining of surrounding material.

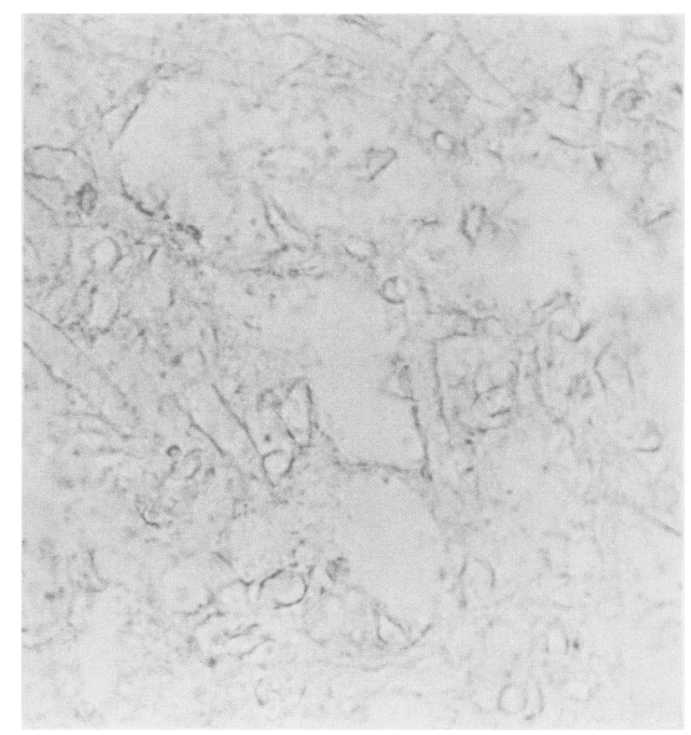

Fig. 5 Hyphae outlined by lectin shown by avidin-biotin method. (Wheat germ agglutinin.) Original magnification $\times$ 170.

The various lectin stains showed considerable dif $\overparen{\varrho}$ ferences in affinity (Table). The hyphae were or clearly, though faintly, stained using the avidin biotin method (Fig. 5), and a semiquantitative assessment of density of staining could be made easily. Lectins labelled with fluorescein gave denser staining of hyphae, but the non-specific background staining tended to be distracting (Fig. 6). Similar background staining was seen on avidin-biotin staining with concanavalin $A$ and, to a lesser degree, with soy bean agglutinin. In both cases hyphae were outlined by negative staining.

The pattern of lectin staining was compared with that obtained with several fungal hyphae from the National Type Collection at Kew (BM Herbertson and RW Stoddart, unpublished data). This showed an affinity pattern similar to that of $\boldsymbol{R}$ oryzae, and there were appreciable differences from the staining patterns of Mortierella wolfii, $R$ arrhizus, Absidia corymbifera, Aspergillus flavus, $A$ fumigatus, $A$ niger, and all of the dermatophytes investigated to date.

\section{Discussion}

The Zygomycetes are a class of fungi usually found in soil, humus, and dung ${ }^{16}$; they cause decay in many kinds of fruit and vegetables ${ }^{17}$ and are occasionally pathogenic in domestic animals. ${ }^{18}$ Spores of various members of the class have been widely detected in 


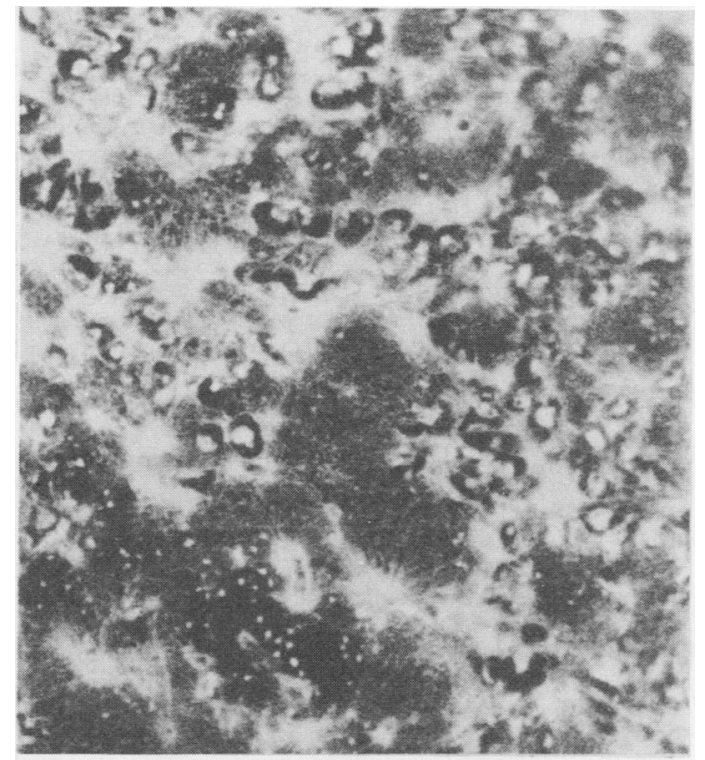

Fig. 6 Fluorescein labelled lectin staining of hyphae. Surrounding fibrinous clot is distractingly stained. (Wheat germ agglutinin, fuorescein isothiocyanate.) Original magnification $\times 170$.

air, including air in a ward in a London teaching hospital. ${ }^{19}$ Skin tests suggest sensitisation in a substantial minority of patients with allergic diseases and in controls, ${ }^{20}$ and sputum from apparently healthy men may be contaminated. ${ }^{21}$

Our failure to diagnose zygomycosis in this case would appear to have been due to a low index of suspicion engendered by the apparent rarity of the disease in this country. Few well documented cases have been reported from the British Isles, ${ }^{22-24}$ though Symmers was able briefly to discuss the findings in "many" cases of British origin. ${ }^{25}$

Authors from several continents have described increases in the number of reported cases of mucormycosis, ${ }^{25-27}$ a change that is partly due to an increase in the incidence of certain of the predisposing factors, partly to greater international mobility, and partly to greater awareness of the condition. ${ }^{25}$ Increasingly aggressive chemotherapy and more widely used antibacterial treatment may also be important.?

Zygomycosis arising in association with erythroleukaemia is rare, presumably because of the rarity of erythroleukaemia, though the association is by no means unknown. ${ }^{28}$ It is not possible to separate the relative importance of disease and treatment in inducing susceptibility to this opportunistic infection, though several cases after renal, ${ }^{29} 30$ cardiac, $^{29}$ and bone marrow transplantation ${ }^{31}$ would suggest that cytotoxic treatment has an important role. Zygomycosis after renal failure without transplantation is less common, and in such cases the patient may also have diabetes mellitus, or at least glucose intolerance induced by steroids. ${ }^{22} 30$

Zygomycosis in patients with leukaemia is particularly associated with periods of neutropenia ${ }^{6}$ and, once established, tends to advance rapidly; blood vessel walls are easily breached, and infarction after thrombosis induced by fungus facilitates the spread of disease. Although mucormycosis is often widespread at the time of detection when leukaemia is the underlying condition, it may start its course in the nose and orbit; this is apparently the second most common portal of entry after the lung in those immunosuppressed following chemotherapy. ${ }^{32}$ Thrombosis of an internal carotid artery, which was a striking feature of this case, is described in at least a third of patients affected cerebrally. ${ }^{33}$

The facility with which the organism spreads once infection is established, coupled with the rarity of the condition, has led to a considerable amount of experimental work aimed at determining what the body's normal defence against mucormycosis might be. It would seem that these defences are multifactorial, with components in both the serum and the phagocytic cell system. ${ }^{34}$ It is also clear that mechanisms exist that inhibit both spore germination and hyphal growth. ${ }^{35}$

Opportunistic infection is often a disaster because it may kill a patient with an otherwise treatable or curable disease. As chemotherapeutic regimens become more potent and rates of remission improve it becomes necessary to guard against a parallel increase in opportunistic infections. The possibility of nosocomial zygomycosis ${ }^{3036}$ makes each case an indicator of potentially similar disease in other patients. Many of the cases reported concern patients whose zygomycosis was discovered only at necropsy, often at a stage when culture was no longer possible. This is a problem that is common in various other opportunistic fungal infections also, and so techniques, such as lectin staining, that offer possible improvements in our ability to recognise and characterise such infections are to be welcomed.

We thank Mr GW Rogers and Mrs J Crosby for photographic help, and Dr CJP Jones and Mr P Ward for technical help.

\section{References}

\footnotetext{
Baker RD. Mucormycosis-a new disease? J Am M Ass 1957; 163: 805-8.

${ }^{2}$ Straatsma BR, Zimmerman LE, Gass JDM. Phycomycosis. A clinicopathologic study of 51 cases. Lab Invest 1962;11:96387.
} 
${ }^{3}$ Lehrer RI, Howard DH, Sypherd PS, Edwards JE, Segal GP, Winston DJ. Mucormycosis. Ann Intern Med 1980;93:93-108.

${ }^{4}$ Pillsbury HC, Fischer ND. Rhinocerebral mucormycosis. Arch Otolaryngol 1977;103:600-4.

s del Real Mora O, Quezada JZ, Mendoza CA, Diaz ES, de Leon SP, Ruiz-Palacois G. Mucormicosis. Informe de 14 cases. Rev Invest Clin 1983;35:237-40.

- Baker RD. Leukopenia and therapy in leukemia as factor predisposing to fatal mycoses, mucormycosis, aspergillosis and cryptococcosis. Am J Clin Pathol 1962;37:358-73.

' Meyer RD, Rosen P, Armstrong D. Phycomycosis complicating leukemia and lymphoma. Ann Intern Med 1972;77:871-9.

${ }^{8}$ McNulty JS. Rhinocerebral mucormycosis: predisposing factors. Laryngoscope 1982;92:1140-3.

' Emmons CW, Binford CH, Utz PJ, Kwon-Chung KJ. Medical mycology, 3rd ed. Philadelphia: Lea and Febiger, 1977:254.

${ }^{10}$ Gordon MA. Nomenclature of fungi pathogenic to man and animals. Sabouraudia 1978;16:312-3.

"Vanbreuseghem R, Gentles JC, Mackenzie DWR, Male O, Mantoviani A. Nomenclature of mycoses. Sabouraudia 1980;18: 78-84.

${ }^{12}$ Webster J. Introduction to fungi. 2nd ed. Cambridge: Cambridge University Press, 1980.

${ }^{13}$ Stoddart RW, Herbertson BM. The use of fluorescein-labelled lectins in the detection and identification of fungi pathogenic for man: a preliminary study. J Med Microbiol 1978;11:31524.

${ }^{14}$ Hsu S-M, Raine L. Versatility of biotin-labelled lectins and avidin-biotin-peroxidase complex for localization of carbohydrate in tissue sections. J Histochem Cytochem 1982;30:15761.

is Hsu S-M, Raine L, Fanger $\mathrm{H}$. The use of avidin antibody and avidin-biotin-peroxidase complex in immunoperoxidase technics. Am J Clin Pathol 1981;75:816-21.

${ }^{16}$ Emmons CW. Natural occurrence of opportunistic fungi. Lab Invest 1962;11:1026-32.

${ }^{17}$ Harter LL, Weimer JL. Decay of various vegetables and fruits by different species of Rhizopus. Phytopathology 1922;12:20512.

${ }^{18}$ Monga DP, Mohapatra LN. A compilation of published reports of mycoses in animals in India. Mycopathologia 1980;72:3-11.

${ }^{14}$ Noble WC, Clayton YM. Fungi in the air of hospital wards. J Gen Microbiol 1963;32:397-402.

${ }^{20}$ Shivpuri DN, Agarwal MK. Studies on the allergenic fungal spores of the Delhi, India, metropolitan area. Clinical aspects. J Allergy 1969;44:204-13.

${ }^{21}$ Comstock GW, Palmer CE, Stone RW, Goodman NL. Fungi in the sputum of normal men. Mycopathologia 1974;54:55-62.

${ }^{22}$ Kurrein F. Cerebral mucormycosis. J Clin Pathol 1954;7:141-4.

${ }^{23}$ Symmers WStC. Silicone mastitis in "topless" waitresses and some other varieties of foreign-oody mastitis. $\mathrm{Br} \mathrm{Med} \mathrm{J}$ 1968;3:19-22.

${ }^{24}$ Helenglass G, Elliott JA, Lucie NP. An unusual presentation of opportunistic mucormycosis. Br Med J 1981;282:108-9.

${ }^{25}$ Symmers WStC. Deep seated fungal infections currently seen in the histopathologic service of a medical school laboratory in Britain. Am J Clin Pathol 1966;46:414-37.

${ }^{26}$ Hotchi M, Okada M, Nasu T. Present state of fungal infections in autopsy cases in Japan. A statistical survey of all autopsy cases during the ten-year period from 1966 to 1975. Am J Clin Pathol 1980;74:410-6.

${ }^{27}$ Marchevsky AM, Bottone EJ, Geller SA, Giger DK. The changing pattern of disease, etiology and diagnosis of mucormycosis Human Pathol 1980;11:457-64.

${ }^{28}$ Rosenberg SW, Lepley JB. Mucromycosis in leukemia. Oral Surg 1982;54:26-32.

${ }^{24}$ Bottone EJ, Weitzman I, Hanna BA. Rhizopus rhizopodiformis: emerging etiological agent of mucormycosis. J Clin Microbiol 1979;9:530-7.

${ }^{30}$ England AC, Weinstein M, Ellner JJ, Ajello L. Two cases of rhinocerebral zygomycosis (mucormycosis) with common epidemiologic and environmental features. Am Rev Respir Dis 1981;124:497-8.

${ }^{31}$ Myskowski PL, Brown AE, Dinsmore R, et al. Mucormycosis following bone marrow transplantation. J Am Acad Dermatol 1983;9:111-5.

${ }^{32}$ Krick JA, Remington JS. Opportunistic invasive fungal infections in patients with leukaemia and lymphoma. Clin Haematol 1976; 5: 249-310.

${ }^{33}$ Landau JW, Newcomer VD. Acute cerebral phycomycosis (mucormycosis). Report of a pediatric patient successfully treated with amphotericin B and cycloheximide and review of the pertinent literature. J Pediatr 1962;61:363-85.

${ }^{34}$ Chinn RYW, Diamond RD. Generation of chemotactic factors by Rhizopus oryzae in the presence and absence of serume relationship to hyphal damage mediated by human neutrophils and effects of hyperglycaemia and ketoacidosis. Infect Immun 1982;38:1123-9.

${ }^{35}$ Waldorf AR, Peter L, Polak A. Mucormycotic infection in mice following prolonged incubation of spores in vivo and the role of spore agglutinating antibodies on spore germination. Sabouraudia 1984;22: 101-8.

${ }^{36}$ Gartenberg G, Bottone EJ, Kensch GT, Weitzman I. Hospitalacquired mucormycosis (Rhizopus rhizopodiformis) of skin and subcutaneous tissue. Epidemiology, mycology and treatment. $N$ Engl J Med 1978;299: 1115-8.

Requests for reprints to: Dr EW Benbow, Department of Pathology, Stopford Building, University of Manchester, Oxford Road, Manchester M13 9PT, England. 